\title{
Erratum
}

\section{Social Inequalities in Health and Closing the Gap in a Generation}

Carme Borrell, Lucia Artazcoz, Maria I. Pasarin and Elia Diez Journal of Public Health Policy (2009) 30, 365. doi:I0.I057/jphp.2009.42

Correction to: Journal of Public Health Policy (2009) 30, I89-194. doi:I0.1057/jphp.2009.I7

In the above article and issue the name of author Maria I. Pasarin was incorrectly published as Mavia I. Pasarin 